\title{
On the regularity of the maximal function of a BV function*
}

\author{
Panu Lahti
}

July 14, 2020

\begin{abstract}
We show that the non-centered maximal function of a BV function is quasicontinuous. We also show that if the non-centered maximal functions of an SBV function is a BV function, then it is in fact a Sobolev function. Using a recent result of Weigt [12], we are in particular able to show that the non-centered maximal function of a set of finite perimeter is a Sobolev function.
\end{abstract}

\section{Introduction}

An open problem that has attracted significant attention in the past two decades is the so-called $W^{1,1}$-problem: is the Hardy-Littlewood maximal function of a Sobolev function $u \in W^{1,1}\left(\mathbb{R}^{d}\right)$ also (locally) in the $W^{1,1}$-class? Typically also a bound $\|\nabla M u\|_{L^{1}\left(\mathbb{R}^{d}\right)} \leq C\|\nabla u\|_{L^{1}\left(\mathbb{R}^{d}\right)}$ is expected to hold. In the case $1<p<\infty$ the analogous result is known to hold, as first shown by Kinnunen [6]. The same is true for the non-centered maximal function, defined by

$$
M u(x):=\sup _{x \in B(z, r)} f_{B(z, r)}|u| d y, \quad x \in \mathbb{R}^{d} .
$$

We will work with this non-centered version that tends to have better regularity than the ordinary Hardy-Littlewood maximal function, in which only balls centered at $x$ are considered. Tanaka [11] gave a positive answer to the $W^{1,1}$-problem in the case $d=1$. Generalizing this to higher dimensions has received significant attention, but results have been achieved only in very special cases. Luiro [10] gave a positive answer to the problem in the case of radial functions, whereas Aldaz and Pérez Lázaro [2] did the same for block-decreasing functions.

In fact, in [2] the authors considered functions $u \in \mathrm{BV}\left(\mathbb{R}^{d}\right)$ rather than just $u \in W^{1,1}\left(\mathbb{R}^{d}\right)$. And apart from the Sobolev regularity, one can consider other continuity properties of the maximal function, but these are also not well understood;

*2020 Mathematics Subject Classification: 42B25, 26B30.

Keywords: function of bounded variation, Sobolev function, non-centered maximal function, quasicontinuity, absolute continuity 
see [1] for some positive results when $d=1$ as well as counterexamples, and [2] for continuity results for block-decreasing BV functions in general dimensions.

In the current paper we show that in general dimensions and for general $u \in$ $\mathrm{BV}\left(\mathbb{R}^{d}\right)$, a very natural continuity property, namely quasicontinuity, can be proven for the maximal function. All definitions will be given in Section 2. Most of the time we will consider the maximal function $M_{\Omega} u$ where one considers balls contained in an open set $\Omega \subset \mathbb{R}^{d}$. After proving some preliminary results in Section 3, we prove the following quasicontinuity result in Section 4.

Theorem 1.1. Let $u \in \mathrm{BV}(\Omega)$. Then $M_{\Omega} u$ is 1-quasicontinuous.

Utilizing this result, in Section 5 we study continuity properties of $M_{\Omega} u$ on lines parallel to coordinate axes. Then in Section 6 we examine absolute continuity on lines and membership in the Sobolev class of the maximal function, proving the following theorem. We say that a BV function is a special function of bounded variation, or SBV function, if the variation measure has no Cantor part.

Theorem 1.2. Let $u \in \operatorname{SBV}\left(\mathbb{R}^{d}\right)$. If $M u \in \mathrm{BV}_{\text {loc }}\left(\mathbb{R}^{d}\right)$, then $M u \in W_{\text {loc }}^{1,1}\left(\mathbb{R}^{d}\right)$.

In a potential breakthrough toward a solution to the $W^{1,1}$-problem, Weigt [12] has shown very recently that for a set of finite perimeter $E \subset \Omega$, we have $M_{\Omega} \mathbb{1}_{E} \in$ $\mathrm{BV}_{\text {loc }}(\Omega)$ such that $\left|D M_{\Omega} \mathbb{1}_{E}\right|(\Omega)$ is at most a constant times $\left|D \mathbb{1}_{E}\right|(\Omega)$. We can utilize this result and go a step further to the desired Sobolev regularity at least in the global case $\Omega=\mathbb{R}^{d}$, as follows.

Theorem 1.3. Let $E \subset \mathbb{R}^{d}$ be a set of finite perimeter. Then $M \mathbb{1}_{E} \in W_{\mathrm{loc}}^{1,1}\left(\mathbb{R}^{d}\right)$ with $\left\|\nabla M \mathbb{1}_{E}\right\|_{L^{1}\left(\mathbb{R}^{d}\right)} \leq C_{d}\left|D \mathbb{1}_{E}\right|\left(\mathbb{R}^{d}\right)$, where $C_{d}$ only depends on the dimension $d$.

Finally, in Sections 7 and 8 we study formulas for the gradient of the maximal function, as well as some further properties in the case $d=1$.

Acknowledgments. The author wishes to thank Julian Weigt for comments and for suggesting the proof of Lemma 3.1. Part of the research for this paper was done while the author was employed at the University of Augsburg.

\section{Notation and definitions}

\subsection{Basic notation}

We will always work in the Euclidean space $\mathbb{R}^{d}, d \geq 1$. We denote the $d$-dimensional Lebesgue measure by $\mathcal{L}^{d}$ and the $s$-dimensional Hausdorff measure by $\mathcal{H}^{s}, s \geq 0$. We denote the characteristic function of a set $E \subset \mathbb{R}^{d}$ by $\mathbb{1}_{E}$.

We write $B(x, r)$ for an open ball in $\mathbb{R}^{d}$ with center $x$ and radius $r$, that is, $\left\{y \in \mathbb{R}^{d}:|y-x|<r\right\}$, and we write $\mathbb{S}^{d-1}$ for the unit sphere in $\mathbb{R}^{d}$, that is, $\{y \in$ $\left.\mathbb{R}^{d}:|y|=1\right\}$. When we consider closed balls, we always specify this by the bar $\bar{B}(x, r)$. 
For a function $u$ we write $u_{+}:=\max \{u, 0\}$ for its positive part, and if it is integrable on some measurable set $D \subset \mathbb{R}^{d}$ of positive and finite Lebesgue measure, we write

$$
f_{D} u(y) d y:=\frac{1}{\mathcal{L}^{d}(D)} \int_{D} u(y) d y
$$

for its mean value on $D$.

We will always denote by $\Omega \subset \mathbb{R}^{d}$ a nonempty open set. The Sobolev space $W^{1,1}(\Omega)$ consists of functions $u \in L^{1}(\Omega)$ whose first weak partial derivatives $D_{k} u$, $k=1, \ldots, d$, belong to $L^{1}(\Omega)$.

The Sobolev 1-capacity of a set $A \subset \mathbb{R}^{d}$ is defined by

$$
\operatorname{Cap}_{1}(A):=\inf \int_{\mathbb{R}^{n}}(|u|+|D u|) d y,
$$

where the infimum is taken over Sobolev functions $u \in W^{1,1}\left(\mathbb{R}^{d}\right)$ satisfying $u \geq 1$ in a neighborhood of $A$. The Sobolev 1-capacity is countably subadditive. Using a cutoff function we find that for every ball $B(x, r)$ with $0<r \leq 1$, we have

$$
\operatorname{Cap}_{1}(B(x, r)) \leq C_{0} r^{d-1}
$$

for a constant $C_{0}$ depending only on $d$.

We say that a function $v$ on $\Omega$ (generally we understand functions to take values in $[-\infty, \infty])$ is 1 -quasicontinuous if for every $\varepsilon>0$ there exists an open set $G \subset \Omega$ such that $\operatorname{Cap}_{1}(G)<\varepsilon$ and $\left.v\right|_{\Omega \backslash G}$ is finite and continuous.

By e.g. [5, Theorem 4.3, Theorem 5.1] we know that for any $A \subset \mathbb{R}^{d}$,

$$
\operatorname{Cap}_{1}(A)=0 \text { if and only if } \mathcal{H}^{d-1}(A)=0 .
$$

The non-centered maximal function of a measurable function $u$ on $\Omega$ is defined by

$$
M_{\Omega} u(x):=\sup _{x \in B(z, r) \subset \Omega} f_{B(z, r)}|u| d y, \quad x \in \Omega .
$$

Sometimes we do not mention $\Omega$ and then it is understood that $\Omega=\mathbb{R}^{d}$, so that $M u:=M_{\mathbb{R}^{d}} u$.

For $\ell \in \mathbb{N}$, we denote by $\mathcal{M}\left(\Omega ; \mathbb{R}^{\ell}\right)$ the Banach space of vector-valued Radon measures $\mu$, equipped with the total variation norm $|\mu|(\Omega)<\infty$, which is defined relative to the Euclidean norm on $\mathbb{R}^{\ell}$. By the Riesz representation theorem, $\mathcal{M}\left(\Omega ; \mathbb{R}^{\ell}\right)$ can be identified with the dual space of $C_{0}\left(\Omega ; \mathbb{R}^{\ell}\right)$ through the duality pairing $\langle\phi, \mu\rangle:=\int_{\Omega} \phi \cdot d \mu:=\sum_{j=1}^{\ell} \int_{\Omega} \phi_{j} d \mu_{j}$. Thus weak* convergence $\mu_{i} \stackrel{*}{\rightarrow} \mu$ in $\mathcal{M}\left(\Omega ; \mathbb{R}^{\ell}\right)$ means $\left\langle\phi, \mu_{i}\right\rangle \rightarrow\langle\phi, \mu\rangle$ for all $\phi \in C_{0}\left(\Omega ; \mathbb{R}^{\ell}\right)$.

We denote the restriction of a measure $\nu$ to a set $A \subset \mathbb{R}^{d}$ by $\nu\llcorner A$, that is,

$$
\nu\left\llcorner A(H):=\nu(A \cap H), \quad H \subset \mathbb{R}^{d} .\right.
$$

For a vector-valued Radon measure $\gamma \in \mathcal{M}\left(\Omega ; \mathbb{R}^{\ell}\right)$ and a positive Radon measure, we can write the Radon-Nikodym decomposition

$$
\gamma=\gamma^{a}+\gamma^{s}=\frac{d \gamma}{d \mu} d \mu+\gamma^{s}
$$

of $\gamma$ with respect to $\mu$, where $\frac{d \gamma}{d \mu} \in L^{1}\left(\Omega, \mu ; \mathbb{R}^{\ell}\right)$. 


\subsection{Functions of bounded variation}

The theory of BV functions presented here can be found e.g. in [3], and we give precise references only for a few key facts. A function $u \in L^{1}(\Omega)$ is a function of bounded variation, denoted $u \in \mathrm{BV}(\Omega)$, if its distributional derivative is an $\mathbb{R}^{d}$-valued Radon measure with finite total variation. This means that there exists a (unique) Radon measure $D u \in \mathcal{M}\left(\Omega ; \mathbb{R}^{d}\right)$ such that for all $\varphi \in C_{c}^{1}(\Omega)$, the integration-by-parts formula

$$
\int_{\Omega} u \frac{\partial \varphi}{\partial y_{j}} d y=-\int_{\Omega} \varphi d(D u)_{j}, \quad j=1, \ldots, d
$$

holds.

If we do not know a priori that a function $u \in L_{\text {loc }}^{1}(\Omega)$ is a BV function, we consider

$$
\operatorname{Var}(u, \Omega):=\sup \left\{\int_{\Omega} u \operatorname{div} \varphi d y, \varphi \in C_{c}^{1}(\Omega),|\varphi| \leq 1\right\} .
$$

If $\operatorname{Var}(u, \Omega)<\infty$, then the Radon measure $D u$ exists and $\operatorname{Var}(u, \Omega)=|D u|(\Omega)$ by the Riesz representation theorem, and $u \in \mathrm{BV}(\Omega)$ provided that $u \in L^{1}(\Omega)$. If $E \subset \mathbb{R}^{d}$ with $\operatorname{Var}\left(\mathbb{1}_{E}, \mathbb{R}^{d}\right)<\infty$, we say that $E$ is a set of finite perimeter.

A fact that we will use many times is that if $u \in \operatorname{BV}_{\text {loc }}(\Omega)$, then also $|u| \in$ $\mathrm{BV}_{\text {loc }}(\Omega)$ with $|D| u||(\Omega) \leq|D u|(\Omega)$.

Let $u$ be a function on $\Omega$. We say that $x \in \Omega$ is a Lebesgue point of $u$ if

$$
\lim _{r \rightarrow 0} f_{B(x, r)}|u(y)-\widetilde{u}(x)| d y=0
$$

for some $\widetilde{u}(x) \in \mathbb{R}$. We denote by $S_{u} \subset \Omega$ the set where this condition fails and call it the approximate discontinuity set.

Given $\nu \in \mathbb{S}^{d-1}$, we define the half-balls

$$
\begin{aligned}
& B_{\nu}^{+}(x, r):=\{y \in B(x, r):\langle y-x, \nu\rangle>0\}, \\
& B_{\nu}^{-}(x, r):=\{y \in B(x, r):\langle y-x, \nu\rangle<0\} .
\end{aligned}
$$

We say that $x \in \Omega$ is an approximate jump point of $u$ if there exist $\nu \in \mathbb{S}^{d-1}$ and distinct numbers $u^{+}(x), u^{-}(x) \in \mathbb{R}$ such that

$$
\lim _{r \rightarrow 0} f_{B_{\nu}^{+}(x, r)}\left|u(y)-u^{+}(x)\right| d y=0
$$

and

$$
\lim _{r \rightarrow 0} f_{B_{\nu}^{-}(x, r)}\left|u(y)-u^{-}(x)\right| d y=0 .
$$

The set of all approximate jump points is denoted by $J_{u}$. We have that $\mathcal{H}^{d-1}\left(S_{u} \backslash\right.$ $\left.J_{u}\right)=0$, see [3, Theorem 3.78]. by

The lower and upper approximate limits of a function $u$ are defined respectively

$$
u^{\wedge}(x):=\sup \left\{t \in \mathbb{R}: \lim _{r \rightarrow 0} \frac{\mathcal{L}^{d}(B(x, r) \cap\{u<t\})}{\mathcal{L}^{d}(B(x, r))}=0\right\}
$$


and

$$
u^{\vee}(x):=\inf \left\{t \in \mathbb{R}: \lim _{r \rightarrow 0} \frac{\mathcal{L}^{d}(B(x, r) \cap\{u>t\})}{\mathcal{L}^{d}(B(x, r))}=0\right\} .
$$

Note that for all $x \in \Omega \backslash S_{u}$, we have $\widetilde{u}(x)=u^{\wedge}(x)=u^{\vee}(x)$. Also, for all $x \in J_{u}$, we have $u^{\wedge}(x)=\min \left\{u^{-}(x), u^{+}(x)\right\}$ and $u^{\vee}(x)=\max \left\{u^{-}(x), u^{+}(x)\right\}$.

We write the Radon-Nikodym decomposition of the variation measure of $u$ into the absolutely continuous and singular parts as $D u=D^{a} u+D^{s} u$. Furthermore, we define the Cantor and jump parts of $D u$ by

$$
D^{c} u:=D^{s} u\left\llcorner\left(\Omega \backslash S_{u}\right), \quad D^{j} u:=D^{s} u\left\llcorner J_{u} .\right.\right.
$$

Since $\mathcal{H}^{d-1}\left(S_{u} \backslash J_{u}\right)=0$ and $|D u|$ vanishes on $\mathcal{H}^{d-1}$-negligible sets, we get the decomposition

$$
D u=D^{a} u+D^{c} u+D^{j} u .
$$

We say that $u \in \mathrm{BV}(\Omega)$ is a special function of bounded variation, and denote $u \in \operatorname{SBV}(\Omega)$, if $\left|D^{c} u\right|(\Omega)=0$.

\subsection{One-dimensional sections of BV functions}

For basic results in the one-dimensional case $d=1$ (with slightly different notation from ours), see [3, Section 3.2]. In this setting, given an open set $\Omega \subset \mathbb{R}$ and $u \in \mathrm{BV}_{\text {loc }}(\Omega)$, we have $J_{u}=S_{u}, J_{u}$ is at most countable, and $D u(\{x\})=0$ for every $x \in \Omega \backslash J_{u}$. For every $x, \widetilde{x} \in \Omega$ in a connected component of $\Omega$, we have

$$
\left|u^{\vee}(\widetilde{x})-u^{\vee}(x)\right| \leq|D u|([x, \widetilde{x}]) .
$$

Thus at every point outside $S_{u}$, the pointwise representative $u^{\wedge}=u^{\vee}=\widetilde{u}$ is continuous. Moreover, $u^{\vee}$ is upper semicontinuous.

In $\mathbb{R}^{d}$, denote by $\pi: \mathbb{R}^{d} \rightarrow \mathbb{R}^{d-1}$ the orthogonal projection onto $\mathbb{R}^{d-1}$ : for $x=$ $\left(x_{1}, \ldots, x_{d}\right) \in \mathbb{R}^{d}$,

$$
\pi\left(\left(x_{1}, \ldots, x_{d}\right)\right):=\left(x_{1}, \ldots, x_{d-1}\right) .
$$

Denote the standard basis vectors by $e_{k}, k=1, \ldots, d$. For an open set $\Omega \subset \mathbb{R}^{d}$, and $u \in \mathrm{BV}(\Omega)$, denote $D_{k} u:=\left\langle D u, e_{k}\right\rangle$. For any fixed $k \in\{1, \ldots, d\}$ - for simplicity we can assume $k=d$ - for every $z \in \pi(\Omega)$ we denote the slices of $\Omega$ at $(z, 0)$ in $e_{d}$-direction by

$$
\Omega_{z}:=\left\{t \in \mathbb{R}:\left(z, t e_{d}\right) \in \Omega\right\} .
$$

We also denote $u_{z}(t):=u(z, t)$ for $z \in \pi(\Omega)$ and $t \in \Omega_{z}$. We know that for $\mathcal{L}^{d-1}$ almost every $z \in \pi(\Omega)$, we have $u_{z} \in \operatorname{BV}\left(\Omega_{z}\right)$ (see [3, Theorem 3.103]) and also, if $u \in \operatorname{SBV}(\Omega)$, then $u_{z} \in \operatorname{SBV}\left(\Omega_{z}\right)$ (see [3, Eq. (3.108)]). On the other hand, if $u_{z}$ is absolutely continuous for almost every $z \in \pi(\Omega)$, and similarly in the other coordinate directions, then $u \in W_{\text {loc }}^{1,1}(\Omega)$. Finally, for $\mathcal{L}^{d-1}$-almost every $z \in \pi(\Omega)$ it holds that

$$
S_{u_{z}}=\left(S_{u}\right)_{z} \quad \text { and } \quad(\widetilde{u})_{z}(t)=\widetilde{u_{z}}(t) \text { for every } t \in \mathbb{R} \backslash S_{u_{z}} \text {, }
$$

see [3, Theorem 3.108]. 


\section{Preliminary results}

In this section we record and prove some preliminary results. Let $\Omega \subset \mathbb{R}^{d}$ always denote an arbitrary nonempty open set.

The following fact is generally well known and used e.g. in [10]. It simply says that in the definition of the non-centered maximal function, the supremum can be taken over balls whose closure contains the point $x$. For the maximal function $M_{\Omega} u$, this fact is not as trivial as it is for the global version $M u=M_{\mathbb{R}^{d}} u$, so we give a short proof.

Lemma 3.1. Let $u$ be a measurable function on $\Omega$. Then we have

$$
M_{\Omega} u(x)=\sup _{x \in \bar{B}(z, r), B(z, r) \subset \Omega} f_{B(z, r)}|u| d y, \quad x \in \Omega .
$$

Proof. Consider a ball $B(z, r) \subset \Omega$ and a point $x \in \Omega \cap \partial B(z, r)$. For some $\delta>0$, we have $B(x, \delta) \subset \Omega$. Let $0<\varepsilon<1 / 3$. The ball $B(z+\varepsilon(x-z),(1-\varepsilon) r)$ is contained in $B(z, r)$. Then clearly for sufficiently small $t \in(0, \varepsilon)$, the ball

$$
B(z+(\varepsilon+t)(x-z),(1-\varepsilon) r)
$$

is contained in $B(z, r) \cup B(x, \delta) \subset \Omega$, and contains $x$ and contains $B(z,(1-3 \varepsilon) r)$. Thus (some of the integrals below could be $+\infty$ )

$$
M_{\Omega} u(x) \geq f_{B(z+(\varepsilon+t)(x-z),(1-\varepsilon) r)}|u| d y \geq \frac{1}{\mathcal{L}^{d}(B(z, r))} \int_{B(z,(1-3 \varepsilon) r)}|u| d y .
$$

Letting $\varepsilon \rightarrow 0$, we obtain

$$
M_{\Omega} u(x) \geq f_{B(z, r)}|u| d y
$$

The following simple property of the non-centered maximal function is crucial for proving the 1-quasicontinuity of $M_{\Omega} u$.

Proposition 3.2. Let $u \in \mathrm{BV}_{\text {loc }}(\Omega)$. Then $M_{\Omega} u(x) \geq u^{\vee}(x)$ for every $x \in \Omega \backslash\left(S_{u} \backslash\right.$ $J_{u}$ ), that is, for $\mathcal{H}^{d-1}$-almost every $x \in \Omega$.

Proof. We obviously have $M_{\Omega} u(x) \geq \widetilde{u}(x)=u^{\vee}(x)$ for every $x \in \Omega \backslash S_{u}$, that is, for Lebesgue points $x$ (recall (2.4)). Assume then that $x \in J_{u}$. Now $u^{\vee}(x)=$ $\max \left\{u^{-}(x), u^{+}(x)\right\}$ (recall (2.5)). Supposing $u^{\vee}(x)=u^{+}(x)$, we obtain using Lemma 3.1 ,

$$
M_{\Omega} u(x) \geq \limsup _{r \rightarrow 0} f_{B(x+(r / 2) \nu, r / 2)} u d y=u^{\vee}(x) .
$$

The case $u^{\vee}(x)=u^{-}(x)$ is similar. The proof is completed by recalling that $\mathcal{H}^{d-1}\left(S_{u} \backslash J_{u}\right)=0$. 
As noted by Aldaz and Pérez Lázaro [1], a BV function need not have any upper semicontinuous representative when $d \geq 2$, which causes difficulties since usually such a representative is used in proving the continuity of $M_{\Omega} u$. However, the following quasi-semicontinuity result given in [4, Theorem 2.5] is a useful substitute. Alternatively, see [9, Theorem 1.1] and [8, Corollary 4.2] for a proof of this result in more general metric spaces.

Theorem 3.3. Let $u \in \mathrm{BV}_{\text {loc }}(\Omega)$ and $\varepsilon>0$. Then there exists an open set $G \subset \Omega$ such that $\operatorname{Cap}_{1}(G)<\varepsilon$ and $\left.u^{\wedge}\right|_{\Omega \backslash G}$ is finite and lower semicontinuous, and $\left.u^{\vee}\right|_{\Omega \backslash G}$ is finite and upper semicontinuous.

The following weak type estimate is well known and a proof can be found e.g. in [7, Lemma 4.3].

Proposition 3.4. Let $u \in \mathrm{BV}\left(\mathbb{R}^{d}\right)$. Then we have for every $t>0$ that

$$
\operatorname{Cap}_{1}\left(\left\{x \in \mathbb{R}^{d}: M u(x)>t\right\}\right) \leq C_{1} \frac{|D u|\left(\mathbb{R}^{d}\right)}{t},
$$

where $C_{1}$ only depends on $d$.

We define auxiliary maximal operators $M_{\Omega}^{R}$ and $M_{\Omega, R}, R>0$, by

$$
M_{\Omega}^{R} u(x):=\sup _{x \in B(z, r) \subset \Omega, r<R} f_{B(z, r)}|u| d y, \quad x \in \Omega,
$$

and

$$
M_{\Omega, R} u(x):=\sup _{x \in B(z, r) \subset \Omega, r \geq R} f_{B(z, r)}|u| d y, \quad x \in \Omega .
$$

Obviously $M_{\Omega} u=\max \left\{M_{\Omega}^{R} u, M_{\Omega, R} u\right\}$. Again if $\Omega=\mathbb{R}^{d}$, we omit it from the notation.

Proposition 3.6. Let $u \in \mathrm{BV}_{\text {loc }}(\Omega)$. Then $\operatorname{Cap}_{1}\left(\left\{x \in \Omega: M_{\Omega} u(x)=\infty\right\}\right)=0$.

Proof. Consider the open sets

$$
\Omega_{j}:=\left\{x \in \Omega: \operatorname{dist}\left(x, \mathbb{R}^{d} \backslash \Omega\right)>2^{-j}\right\}, \quad j \in \mathbb{N} .
$$

Now $\bigcup_{j=1}^{\infty} \Omega_{j}=\Omega$. Choose cutoff functions $\eta_{j} \in C_{c}^{\infty}(\Omega)$ with $0 \leq \eta_{j} \leq 1$ in $\mathbb{R}^{d}$ and $\eta_{j}=1$ in $\Omega_{j}$. Now $\eta_{j} u \in \mathrm{BV}\left(\mathbb{R}^{d}\right)$ and so by Proposition 3.4,

$$
\operatorname{Cap}_{1}\left(\left\{x \in \Omega_{j}: M_{\Omega}^{2^{-2 j}} u(x)=\infty\right\}\right) \leq \operatorname{Cap}_{1}\left(\left\{x \in \Omega_{j}: M\left(\eta_{j+1} u\right)(x)=\infty\right\}\right)=0 .
$$

On the other hand, since $u \in L^{1}(\Omega)$, clearly $M_{\Omega, 2^{-2 j}} u(x)<\infty$ for every $x \in \Omega$. In

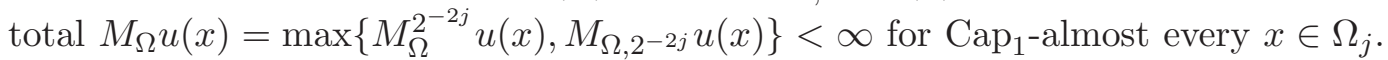
Since $\bigcup_{j=1}^{\infty} \Omega_{j}=\Omega$, we obtain the result.

The following fact is well known; for a proof covering the case $u \in L^{1}\left(\mathbb{R}^{d}\right)$ see e.g. [10, Proposition 3.2], while the case $u \in L^{\infty}\left(\mathbb{R}^{d}\right)$ follows by a slight modification. This result does not necessarily hold for $M_{\Omega} u$ in an open set $\Omega$, which is why we formulate some of the main results of this paper only in the global case $\Omega=\mathbb{R}^{d}$. 
Proposition 3.7. Let $u \in L^{1}\left(\mathbb{R}^{d}\right)$ (resp. $u \in L^{\infty}\left(\mathbb{R}^{d}\right)$ ), and let $R>0$. Then $M_{R} u$ is Lipschitz with constant depending only on $d, R$, and $\|u\|_{L^{1}\left(\mathbb{R}^{d}\right)}$ (resp. $\|u\|_{L^{\infty}\left(\mathbb{R}^{d}\right)}$ ).

The following result proven in [8, Lemma 3.5] is our key tool for handling the exceptional set of quasi (semi)continuity.

Lemma 3.8. Let $G \subset \mathbb{R}^{d}$ and $\varepsilon>0$. Then there exists an open set $U \supset G$ with $\operatorname{Cap}_{1}(U) \leq C_{2} \operatorname{Cap}_{1}(G)+\varepsilon$ such that

$$
\frac{\mathcal{L}^{d}(B(x, r) \cap G)}{\mathcal{L}^{d}(B(x, r))} \rightarrow 0 \quad \text { as } r \rightarrow 0
$$

uniformly for $x \in \mathbb{R}^{d} \backslash U$. Here $C_{2}$ depends only on $d$.

We will need the following version.

Lemma 3.9. Let $u \in \mathrm{BV}_{\text {loc }}(\Omega)$, let $G \subset \Omega$, and let $\varepsilon>0$. Then there exists an open set $U \supset G$ such that $\operatorname{Cap}_{1}(U) \leq C_{2} \operatorname{Cap}_{1}(G)+\varepsilon$ and

$$
\frac{1}{\mathcal{L}^{d}(B(x, r))} \int_{B(x, r) \cap G}|u| d y \rightarrow 0 \quad \text { as } r \rightarrow 0
$$

locally uniformly for $x \in \Omega \backslash U$. Here $C_{2}$ is the same constant as in Lemma 3.8.

Proof. We have $|u| \in \mathrm{BV}_{\text {loc }}(\Omega)$, and so we can assume that $u$ is nonnegative. By Lemma 3.8, we find an open set $W \supset G$ such that $\operatorname{Cap}_{1}(W) \leq C_{2} \operatorname{Cap}_{1}(G)+\varepsilon / 2$ and

$$
\frac{\mathcal{L}^{d}(B(x, r) \cap G)}{\mathcal{L}^{d}(B(x, r))} \rightarrow 0 \quad \text { as } r \rightarrow 0
$$

uniformly for $x \in \mathbb{R}^{d} \backslash W$. Let

$$
\Omega_{j}:=\left\{x \in B(0, j): \operatorname{dist}\left(x, \mathbb{R}^{d} \backslash \Omega\right)>1 / j\right\}, \quad j \in \mathbb{N},
$$

so that $\Omega=\bigcup_{j=1}^{\infty} \Omega_{j}$. Note that we have by the Sobolev embedding

$$
u \in \mathrm{BV}_{\text {loc }}(\Omega) \subset L_{\text {loc }}^{d /(d-1)}(\Omega) \subset L^{d /(d-1)}\left(\Omega_{j}\right) \quad \text { for every } j \in \mathbb{N} .
$$

Choose numbers $\alpha_{j}>0, j=0,1, \ldots$, such that $\alpha_{j+1} \geq 2 \alpha_{j}$ and

$$
\sum_{j=1}^{\infty}\left(\int_{\Omega_{j+1}}\left(u-\alpha_{j-1}\right)_{+}^{d /(d-1)} d y\right)^{(d-1) / d}<\frac{\varepsilon}{5^{d} C_{0}} .
$$

Next take a sequence $\beta_{j} \searrow 0, \beta_{j} \leq 1$, such that still

$$
\sum_{j=1}^{\infty} \frac{1}{\beta_{j}}\left(\int_{\Omega_{j+1}}\left(u-\alpha_{j-1}\right)_{+}^{d /(d-1)} d y\right)^{(d-1) / d}<\frac{\varepsilon}{5^{d} C_{0}} .
$$

Define the sets

$$
E_{j}:=\left\{x \in \Omega: u(x) \geq \alpha_{j}\right\} .
$$


Then define the sets

$$
\begin{aligned}
& A_{j}:=\left\{x \in \Omega_{j}: \frac{1}{\mathcal{L}^{d}(B(x, r))} \int_{B(x, r) \cap E_{j}} u d y>\beta_{j}\right. \\
&\left.\quad \text { for some } 0<r \leq 1 / 5 \text { with } B(x, r) \subset \Omega_{j+1}\right\} .
\end{aligned}
$$

Consider $j \in \mathbb{N}$ and $x \in A_{j}$. For some $0<r_{x} \leq 1 / 5$, we have $B\left(x, r_{x}\right) \subset \Omega_{j+1}$ and

$$
\frac{1}{\mathcal{L}^{d}\left(B\left(x, r_{x}\right)\right)} \int_{B\left(x, r_{x}\right)}\left(u-\alpha_{j-1}\right)_{+} d y \geq \frac{1}{2} \frac{1}{\mathcal{L}^{d}\left(B\left(x, r_{x}\right)\right)} \int_{B\left(x, r_{x}\right) \cap E_{j}} u d y>\frac{\beta_{j}}{2},
$$

and so by Hölder's inequality

$$
\frac{1}{r_{x}^{d-1}}\left(\int_{B\left(x, r_{x}\right)}\left(u-\alpha_{j-1}\right)_{+}^{d /(d-1)} d y\right)^{(d-1) / d}>\frac{\beta_{j}}{2} .
$$

Now $\left\{B\left(x, r_{x}\right)\right\}_{x \in A_{j}}$ is a covering of $A_{j}$. By the 5 -covering theorem, we find a countable collection of pairwise disjoint balls $\left\{B\left(x_{k}, r_{k}\right)\right\}_{k=1}^{\infty}$ such that $A_{j} \subset \bigcup_{k=1}^{\infty} B\left(x_{k}, 5 r_{k}\right)$. Now we have by $(2.1)$, and by using the triangle inequality for the $L^{q /(q-1)}$-norm,

$$
\begin{aligned}
\operatorname{Cap}_{1}\left(A_{j}\right) & \leq \sum_{k=1}^{\infty} \operatorname{Cap}_{1}\left(B\left(x_{k}, 5 r_{k}\right)\right) \\
& \leq 5^{d-1} C_{0} \sum_{k=1}^{\infty} r_{k}^{d-1} \\
& \leq \frac{2 \times 5^{d-1} C_{0}}{\beta_{j}} \sum_{k=1}^{\infty}\left(\int_{B\left(x_{k}, r_{k}\right)}\left(u-\alpha_{j-1}\right)_{+}^{d /(d-1)} d y\right)^{(d-1) / d} \\
& \leq \frac{2 \times 5^{d-1} C_{0}}{\beta_{j}}\left(\int_{\Omega_{j+1}}\left(u-\alpha_{j-1}\right)_{+}^{d /(d-1)} d y\right)^{(d-1) / d} .
\end{aligned}
$$

Now recalling (3.11), we get

$$
\operatorname{Cap}_{1}\left(W \cup \bigcup_{j=1}^{\infty} A_{j}\right) \leq \operatorname{Cap}_{1}(W)+\sum_{j=1}^{\infty} \operatorname{Cap}_{1}\left(A_{j}\right)<C_{2} \operatorname{Cap}_{1}(G)+\frac{\varepsilon}{2}+\frac{\varepsilon}{2} .
$$

Finally, take an open set

$$
U \supset W \cup \bigcup_{j=1}^{\infty} A_{j}
$$

with $\operatorname{Cap}_{1}(U)<C_{2} \operatorname{Cap}_{1}(G)+\varepsilon$.

Fix $\delta>0$. Take $j_{0} \in \mathbb{N}$ sufficiently large that $j_{0} \geq 1 / \delta$ and $\beta_{j_{0}}<\delta / 2$. Using (3.10), take $0<R \leq 1 / 5$ such that

$$
\frac{\mathcal{L}^{d}(G \cap B(x, r))}{\mathcal{L}^{d}(B(x, r))}<\frac{\delta}{2 \alpha_{j_{0}}} \quad \text { for all } x \in \mathbb{R}^{d} \backslash U \text { and } 0<r \leq R .
$$


Thus for all

$$
x \in\left\{y \in B(0,1 / \delta): \operatorname{dist}\left(y, \mathbb{R}^{d} \backslash \Omega\right)>\delta\right\} \backslash U \subset \Omega_{j_{0}} \backslash U
$$

and $0<r \leq \min \left\{R, \operatorname{dist}\left(\Omega_{j_{0}}, \mathbb{R}^{d} \backslash \Omega_{j_{0}+1}\right)\right\}$, we have

$$
\begin{aligned}
& \frac{1}{\mathcal{L}^{d}(B(x, r))} \int_{G \cap B(x, r)} u d y \\
& \quad \leq \frac{1}{\mathcal{L}^{d}(B(x, r))} \int_{G \cap B(x, r) \backslash E_{j_{0}}} u d y+\frac{1}{\mathcal{L}^{d}(B(x, r))} \int_{B(x, r) \cap E_{j_{0}}} u d y \\
& \quad<\frac{\delta}{2 \alpha_{j_{0}}} \alpha_{j_{0}}+\beta_{j_{0}} \leq \frac{\delta}{2}+\frac{\delta}{2}=\delta .
\end{aligned}
$$

Since $\delta>0$ was arbitrary, this proves the local uniform convergence in the set $\Omega \backslash U$.

\section{Quasicontinuity}

In this section we prove that the non-centered maximal function of a $\mathrm{BV}$ function is 1-quasicontinuous. As before, $\Omega \subset \mathbb{R}^{d}$ is an arbitrary nonempty open set.

The following theorem is Theorem 1.1 in a slightly more general form.

Theorem 4.1. Let $u \in \mathrm{BV}_{\text {loc }}(\Omega) \cap L^{1}(\Omega)$ or $u \in \mathrm{BV}_{\text {loc }}\left(\mathbb{R}^{d}\right)$. Then $M_{\Omega} u$ is 1quasicontinuous.

Proof. First assume that $u \in \mathrm{BV}_{\text {loc }}(\Omega) \cap L^{1}(\Omega)$. Then also $|u| \in \operatorname{BV}_{\text {loc }}(\Omega) \cap L^{1}(\Omega)$, and so we can assume that $u$ is nonnegative.

Fix $\varepsilon>0$. By Theorem 3.3 we find an open set $G \subset \Omega$ such that $\operatorname{Cap}_{1}(G)<\varepsilon / C_{2}$ and $\left.u^{\vee}\right|_{\Omega \backslash G}$ is upper semicontinuous. Since $\operatorname{Cap}_{1}\left(S_{u} \backslash J_{u}\right)=\mathcal{H}^{d-1}\left(S_{u} \backslash J_{u}\right)=0$ (recall $(2.2))$ and

$$
\operatorname{Cap}_{1}\left(\left\{x \in \Omega: M_{\Omega} u(x)=\infty\right\}\right)=0
$$

by Proposition 3.6, we can assume that $G \supset\left\{x \in \Omega: M_{\Omega} u(x)=\infty\right\} \cup\left(S_{u} \backslash J_{u}\right)$. Then by Lemma 3.9, we can take an open set $U \supset G$ such that $\operatorname{Cap}_{1}(U)<\varepsilon$ and

$$
\frac{1}{\mathcal{L}^{d}(B(x, r))} \int_{B(x, r) \cap G} u d y \rightarrow 0 \quad \text { as } r \rightarrow 0
$$

locally uniformly for $x \in \Omega \backslash U$. Since $\left.M_{\Omega} u\right|_{\Omega \backslash U}$ is finite and lower semicontinuous, it is sufficient to prove upper semicontinuity. Fix $x \in \Omega \backslash U$. Take a sequence $x_{j} \rightarrow x$, $x_{j} \in \Omega \backslash U$, such that

$$
\lim _{j \rightarrow \infty} M_{\Omega} u\left(x_{j}\right)=\limsup _{\Omega \backslash U \ni y \rightarrow x} M_{\Omega} u(y) .
$$

(At this stage we cannot exclude the possibility that the lim sup is $\infty$.) Now we only need to show that $M_{\Omega} u(x) \geq \lim _{j \rightarrow \infty} M_{\Omega} u\left(x_{j}\right)$. 
We find "almost optimal" balls $B\left(x_{j}^{*}, r_{j}\right)$ in the sense that

$$
\lim _{j \rightarrow \infty} M_{\Omega} u\left(x_{j}\right)=\lim _{j \rightarrow \infty} f_{B\left(x_{j}^{*}, r_{j}\right)} u d y,
$$

with $x_{j} \in B\left(x_{j}^{*}, r_{j}\right) \subset \Omega$. Since $u \in L^{1}(\Omega)$, we can assume that the radii $r_{j}$ are uniformly bounded. Now we consider two cases.

Case 1. Suppose that by passing to a subsequence (not relabeled), we have $r_{j} \rightarrow 0$. Fix $\delta>0$. By the upper semicontinuity of $\left.u^{\vee}\right|_{\Omega \backslash G}$, for some $r>0$ we have $B(x, r) \subset \Omega$ and

$$
u^{\vee}(x) \geq \sup _{B(x, r) \backslash G} u^{\vee}-\delta .
$$

Note also that for sufficiently large $j \in \mathbb{N}$, we have $B\left(x_{j}^{*}, r_{j}\right) \subset B(x, r)$. Thus, using Proposition 3.2 (recall that $G \supset S_{u} \backslash J_{u}$ ), we get for large $j \in \mathbb{N}$

$$
\begin{aligned}
M_{\Omega} u(x) & \geq u^{\vee}(x) \\
& \geq \sup _{B(x, r) \backslash G} u^{\vee}-\delta \quad \text { by }(4.4) \\
& \geq \frac{1}{\mathcal{L}^{d}\left(B\left(x_{j}^{*}, r_{j}\right)\right)} \int_{B\left(x_{j}^{*}, r_{j}\right) \backslash G} u d y-\delta \quad \text { since } B\left(x_{j}^{*}, r_{j}\right) \subset B(x, r) \\
& =\frac{1}{\mathcal{L}^{d}\left(B\left(x_{j}^{*}, r_{j}\right)\right)} \int_{B\left(x_{j}^{*}, r_{j}\right)} u d y-\frac{1}{\mathcal{L}^{d}\left(B\left(x_{j}^{*}, r_{j}\right)\right)} \int_{B\left(x_{j}^{*}, r_{j}\right) \cap G} u d y-\delta \\
& \geq f_{B\left(x_{j}^{*}, r_{j}\right)} u d y-\frac{2^{d}}{\mathcal{L}^{d}\left(B\left(x_{j}, 2 r_{j}\right)\right)} \int_{B\left(x_{j}, 2 r_{j}\right) \cap G} u d y-\delta .
\end{aligned}
$$

Now by (4.2) and (4.3), we get

$$
M_{\Omega} u(x) \geq \lim _{j \rightarrow \infty} M_{\Omega} u\left(x_{j}\right)-\delta,
$$

so that letting $\delta \rightarrow 0$, we obtain the desired inequality.

Case 2. The other alternative is that passing to a subsequence (not relabeled), we have $r_{j} \rightarrow r \in(0, \infty)$. Passing to a further subsequence (not relabeled), the vectors $x_{j}^{*}-x_{j}$ (since they have length at most $r_{j}$ ) converge to some $v \in \mathbb{R}^{d}$. Now for $x^{*}:=x+v$ we have $\mathbb{1}_{B\left(x_{j}^{*}, r_{j}\right)} \rightarrow \mathbb{1}_{B\left(x^{*}, r\right)}$ in $L^{1}\left(\mathbb{R}^{d}\right)$, with $x \in \bar{B}\left(x^{*}, r\right)$ and $B\left(x^{*}, r\right) \subset \Omega$. In this case we have by Lemma 3.1 that

$$
M_{\Omega} u(x) \geq f_{B\left(x^{*}, r\right)} u d y=\lim _{j \rightarrow \infty} f_{B\left(x_{j}^{*}, r_{j}\right)} u d y=\lim _{j \rightarrow \infty} M_{\Omega} u\left(x_{j}\right) .
$$

This completes the proof in the case $u \in \mathrm{BV}_{\text {loc }}(\Omega) \cap L^{1}(\Omega)$.

Now consider the case $u \in \mathrm{BV}_{\text {loc }}\left(\mathbb{R}^{d}\right)$. The proof is the same, except that now we need to consider a third case.

Case 3. Suppose that passing to a subsequence (not relabeled), we have $r_{j} \rightarrow \infty$. Then since $B\left(x_{j}^{*}, r_{j}+1\right) \ni x$ for all large $j \in \mathbb{N}$, we get

$$
M u(x) \geq \limsup _{j \rightarrow \infty} f_{B\left(x_{j}^{*}, r_{j}+1\right)} u d y \geq \limsup _{j \rightarrow \infty} f_{B\left(x_{j}^{*}, r_{j}\right)} u d y=\lim _{j \rightarrow \infty} M u\left(x_{j}\right) .
$$


This completes the proof.

Aldaz and Pérez Lázaro [2] showed that the maximal function of a block-decreasing $\mathrm{BV}$ function is continuous at every point outside a $\mathcal{H}^{d-1}$-negligible set. Such a continuity property is somewhat stronger than 1-quasicontinuity; recall that $\mathrm{Cap}_{1}$ and $\mathcal{H}^{d-1}$ have the same null sets. The following simple example shows that for general BV functions we cannot have such a stronger continuity property, demonstrating that quasicontinuity seems to be the correct concept to consider.

Example 4.5. Take an enumeration of all the points on the plane with rational coordinates $\left\{q_{j}\right\}_{j=1}^{\infty}$ and define the "enlarged rationals"

$$
E:=\bigcup_{j=1}^{\infty} B\left(q_{j}, 2^{-j}\right)
$$

Clearly $\mathcal{L}^{2}(E) \leq \pi / 2$. By lower semicontinuity and subadditivity we have (recall $(2.3))$

$$
\operatorname{Var}\left(\mathbb{1}_{E}, \mathbb{R}^{2}\right) \leq \sum_{j=1}^{\infty} \operatorname{Var}\left(\mathbb{1}_{B\left(q_{j}, 2^{-j}\right)}, \mathbb{R}^{2}\right)=2 \pi \sum_{j=1}^{\infty} 2^{-j}=2 \pi
$$

Thus $\mathbb{1}_{E} \in \mathrm{BV}\left(\mathbb{R}^{2}\right)$. We have $M \mathbb{1}_{E}(x)<1$ for every $x \in \mathbb{R}^{2}$ with $\mathbb{1}_{E}^{\vee}(x)=0$. However, for every such $x$ there is a sequence of points $x_{j} \rightarrow x$ such that $x_{j} \in E$ for every $j \in \mathbb{N}$. Thus $M \mathbb{1}_{E}\left(x_{j}\right)=1$ for every $j \in \mathbb{N}$. Hence $M \mathbb{1}_{E}$ is discontinuous at every point in the set $\left\{x \in \mathbb{R}^{2}: \mathbb{1}_{E}^{\vee}(x)=0\right\}$, which has even infinite Lebesgue measure.

\section{Continuity and Lusin property on lines}

In this section we prove that the non-centered maximal function of an SBV function, when restricted to almost every line parallel to a coordinate axis, is continuous and has the Lusin property. The Lusin property for a function $v$ defined on $V \subset \mathbb{R}$ states that

$$
\text { if } N \subset V \text { with } \mathcal{L}^{1}(N)=0 \text {, then } \mathcal{L}^{1}(v(N))=0 .
$$

First we prove this kind of property in the following form. Recall that $S_{u}$ denotes the set of non-Lebesgue points of $u$, and that $\widetilde{u}$ is the Lebesgue representative of $u$.

Lemma 5.2. Let $V \subset \mathbb{R}$ be open and let $u \in \mathrm{BV}_{\text {loc }}(V)$. If $N \subset V \backslash S_{u}$ with $|D u|(N)=0$, then

$$
\mathcal{L}^{1}(\widetilde{u}(N))=0
$$

Proof. The claim is equivalent with $\mathcal{L}^{1}\left(u^{\vee}(N)\right)=0$; we will work with the everywhere defined representative $u^{\vee}$ since some of the points that we examine may be in the jump set $S_{u}$. Fix $\varepsilon>0$. We can take an open set $U$ with $N \subset U \subset V$ and $|D u|(U)<\varepsilon$. For every $x \in N$, we can choose an arbitrarily short compact 
interval $I \ni x$ contained in $U$. Consider the collection of intervals (understood to be nondegenerate, i.e. consisting of more than one point)

$$
\mathcal{I}:=\{I: x \in I \subset U, x \in N\} .
$$

These form a covering of $N$. Let

$$
H:=\left\{h \in \mathbb{R}: \text { for some } I \in \mathcal{I}, u^{\vee}(I)=\{h\}\right\} .
$$

The set $H$ can be at most countable, since the intervals $I$ are nondegenerate. Now the collection of intervals

$$
\mathcal{J}:=\left\{\left[\inf u^{\vee}(I), \sup u^{\vee}(I)\right]: I \in \mathcal{I}, u^{\vee} \text { is not constant on } I\right\}
$$

is a covering of $u^{\vee}(N) \backslash H$. It is a fine covering, since every $x \in N \subset V \backslash S_{u}$ is a point of continuity of $u^{\vee}$ (recall (2.7)). By Vitali's covering theorem, there exists a countable collection of disjoint intervals $\left\{J_{j}\right\}_{j=1}^{\infty}$ selected from $\mathcal{J}$ such that

$$
\mathcal{L}^{1}\left(u^{\vee}(N) \backslash \bigcup_{j=1}^{\infty} J_{j}\right)=0 .
$$

For every $J_{j}$, there exists $I_{j}$ such that $J_{j}=\left[\inf u^{\vee}\left(I_{j}\right), \sup u^{\vee}\left(I_{j}\right)\right]$. Then by $(2.7)$,

$$
\mathcal{L}^{1}\left(u^{\vee}(N)\right) \leq \sum_{j=1}^{\infty} \mathcal{L}^{1}\left(J_{j}\right) \leq \sum_{j=1}^{\infty}|D u|\left(I_{j}\right) \leq|D u|(U)<\varepsilon .
$$

Since $\varepsilon>0$ was arbitrary, the result follows.

Define

$$
H_{u}:=\left\{x \in \Omega: M_{\Omega} u(x)>|u|^{\vee}(x)\right\} .
$$

Recall that we denote by $\pi: \mathbb{R}^{d} \rightarrow \mathbb{R}^{d-1}$ the orthogonal projection onto $\mathbb{R}^{d-1}$ : for $x=\left(x_{1}, \ldots, x_{d}\right) \in \mathbb{R}^{d}$,

$$
\pi\left(\left(x_{1}, \ldots, x_{d}\right)\right):=\left(x_{1}, \ldots, x_{d-1}\right) .
$$

Proposition 5.3. Let $A \subset \mathbb{R}^{d}$. Then

$$
2 \mathcal{H}^{d-1}(\pi(A)) \leq \operatorname{Cap}_{1}(A) \text {. }
$$

Proof. Consider $u \in W^{1,1}\left(\mathbb{R}^{d}\right)$ with $u \geq 1$ in a neighborhood of $A$. Then on almost every line $l$ in the $d$ th coordinate direction intersecting $A$, we have

$$
\int_{l}\left|\frac{d u}{d x_{d}}\right| d s \geq 2 .
$$

Integrating over $\mathbb{R}^{d-1}$, we get

$$
\int_{\mathbb{R}^{d}}|\nabla u| d x \geq 2 \mathcal{H}^{d-1}(\pi(A)) .
$$

Thus $\|u\|_{W^{1,1}\left(\mathbb{R}^{d}\right)} \geq 2 \mathcal{H}^{d-1}(\pi(A))$ and we get the result by taking infimum over all such $u$. 
Recall from Section 2.2 that a BV function $u$ is in the SBV class if $\left|D^{c} u\right|(\Omega)=0$.

Theorem 5.4. Let $u \in \mathrm{BV}_{\text {loc }}(\Omega) \cap L^{1}(\Omega)$ or $u \in \mathrm{BV}_{\text {loc }}\left(\mathbb{R}^{d}\right)$. Then $M_{\Omega} u$ is continuous on almost every line parallel to a coordinate axis.

If $u \in \mathrm{SBV}_{\text {loc }}\left(\mathbb{R}^{d}\right) \cap L^{1}\left(\mathbb{R}^{d}\right)$ or $u \in \mathrm{SBV}_{\text {loc }}\left(\mathbb{R}^{d}\right) \cap L^{\infty}\left(\mathbb{R}^{d}\right)$, then $M u$ also has the Lusin property on almost every line parallel to a coordinate axis.

Proof. Again we can assume that $u \geq 0$. First we prove the continuity on lines. Fix $\varepsilon>0$. By Theorems 3.3 and 4.1, we can take an open set $G \subset \Omega$ such that $\operatorname{Cap}_{1}(G)<\varepsilon / C_{2}$ and $\left.u^{\vee}\right|_{\Omega \backslash G}$ is finite and upper semicontinuous, and $\left.M_{\Omega} u\right|_{\Omega \backslash G}$ is finite and continuous. Since $\operatorname{Cap}_{1}\left(S_{u} \backslash J_{u}\right)=\mathcal{H}^{d-1}\left(S_{u} \backslash J_{u}\right)=0$ (recall (2.2)), we can also assume that $G \supset S_{u} \backslash J_{u}$. By Lemma 3.9 we can take an open set $U \supset G$ such that $\operatorname{Cap}_{1}(U)<\varepsilon$ and

$$
\frac{1}{\mathcal{L}^{d}(B(x, r))} \int_{G \cap B(x, r)} u d y \rightarrow 0 \quad \text { as } r \rightarrow 0
$$

locally uniformly for $x \in \Omega \backslash U$. We wish to study the behavior of $M_{\Omega} u$ in $H_{u} \backslash U$. Consider $x_{0} \in H_{u} \backslash U$. We have $\alpha:=M_{\Omega} u\left(x_{0}\right) \in(0, \infty)$. Let $\delta:=\alpha-u^{\vee}\left(x_{0}\right)>0$. By upper semicontinuity of $\left.u^{\vee}\right|_{\Omega \backslash G}$, for some $R_{1}>0$ we have

$$
u^{\vee}(x)<\alpha-\frac{3 \delta}{4}
$$

for all $x \in B\left(x_{0}, R_{1}\right) \backslash G$. By lower semicontinuity of the maximal function, there exists $R_{2}>0$ such that $M_{\Omega} u(x)>\alpha-\delta / 4$ for all $x \in B\left(x_{0}, R_{2}\right)$. Moreover, by the choice of the set $U$, there exists $R_{3}>0$ such that for all $x \in B\left(x_{0}, R_{3}\right) \backslash U$ we have

$$
\frac{1}{\mathcal{L}^{d}(B(x, s))} \int_{G \cap B(x, s)} u d y \leq \frac{\delta}{2^{d+1}} \quad \text { for all } 0<s<R_{3} .
$$

Let $R:=\min \left\{R_{1}, R_{2}, R_{3}\right\} / 4$. Now consider any $x \in B\left(x_{0}, R\right) \backslash U$, and any ball $B(z, r) \ni x$ with $r \in(0, R)$. We have

$$
\begin{aligned}
\frac{1}{\mathcal{L}^{d}(B(z, r))} \int_{B(z, r)} u d y & =\frac{1}{\mathcal{L}^{d}(B(z, r))} \int_{B(z, r) \cap G} u d y+\frac{1}{\mathcal{L}^{d}(B(z, r))} \int_{B(z, r) \backslash G} u d y \\
& \leq \frac{2^{d}}{\mathcal{L}^{d}(B(x, 2 r))} \int_{B(x, 2 r) \cap G} u d y+\alpha-\frac{3 \delta}{4} \quad \text { by }(5.5) \\
& \leq \frac{\delta}{2}+\alpha-\frac{3 \delta}{4} \quad \text { by }(5.6) \\
& =\alpha-\frac{\delta}{4} .
\end{aligned}
$$

On the other hand, we had $M_{\Omega} u(x)>\alpha-\delta / 4$ for all $x \in B\left(x_{0}, R\right)$. Recalling the definition (3.5), we have

$$
M_{\Omega} u(x)=M_{\Omega, R} u(x)
$$

for every $x \in B\left(x_{0}, R\right) \backslash U$. 
Now we examine the behavior of $M_{\Omega} u$ on lines. Recall the notation and results from Section 2.3. Without loss of generality we can consider lines parallel to the $d:$ th coordinate axis. Recall that $\pi$ denotes the orthogonal projection onto $\mathbb{R}^{d-1}$. By Proposition 5.3 we know that

$$
\mathcal{H}^{d-1}(\pi(U)) \leq \operatorname{Cap}_{1}(U)<\varepsilon .
$$

Thus it is enough to consider a line not intersecting $U$. In other words, consider a fixed $z \in \pi(\Omega) \backslash \pi(U)$ and then consider the line $(z, t), t \in \mathbb{R}$. Since we know that $\left.M_{\Omega} u\right|_{\Omega \backslash U}$ is continuous, $t \mapsto M_{\Omega} u(z, t)$ is continuous, proving the first claim.

Now suppose $u \in \operatorname{SBV}_{\text {loc }}\left(\mathbb{R}^{d}\right) \cap L^{1}\left(\mathbb{R}^{d}\right)$ or $u \in \operatorname{SBV}_{\text {loc }}\left(\mathbb{R}^{d}\right) \cap L^{\infty}\left(\mathbb{R}^{d}\right)$. The function $u_{z}$ is in the class $\operatorname{SBV}_{\text {loc }}(\mathbb{R})$ for almost every $z \in \mathbb{R}^{d-1}$, and so we can also assume that $u_{z} \in \operatorname{SBV}_{\text {loc }}(\mathbb{R})$. We can further assume that $\left(J_{u}\right)_{z}$ is at most countable; this follows from the fact that $J_{u}$ is countably $d-1$-rectifiable, and from the coarea formula, see [3, Theorem 2.93].

Let $N \subset \mathbb{R}$ with zero 1-dimensional Lebesgue measure. We have

$$
M u(z, N)=M u\left((\{z\} \times N) \cap H_{u}\right) \cup M u\left((\{z\} \times N) \backslash H_{u}\right) .
$$

Here the first set has zero one-dimensional Lebesgue measure by the fact that $\left.M u\right|_{H_{u} \backslash U}$ is locally Lipschitz, which is given by (5.7) and Proposition 3.7. For the second set we have, recalling that $U \supset S_{u} \backslash J_{u}$,

$$
\begin{aligned}
M u\left((\{z\} \times N) \backslash H_{u}\right) & \subset M u\left((\{z\} \times N) \backslash\left(H_{u} \cup S_{u}\right)\right) \cup M u\left((\{z\} \times N) \cap J_{u}\right) \\
& =\widetilde{u}\left((\{z\} \times N) \backslash\left(H_{u} \cup S_{u}\right)\right) \cup M u\left((\{z\} \times N) \cap J_{u}\right) \\
& \subset \widetilde{u_{z}}\left(z, N \backslash S_{u_{z}(\cdot)}\right) \cup M u\left((\{z\} \times N) \cap J_{u}\right) .
\end{aligned}
$$

by (2.8) (which we can assume to hold by discarding another $\mathcal{L}^{d-1}$-negligible set). Here the first set has zero measure since $u_{z} \in \operatorname{SBV}_{\text {loc }}\left(\Omega_{z}\right)$ and so $\left|D u_{z}\right|\left(N \backslash S_{u_{z}(\cdot)}\right)=$ 0 , and then we can use Lemma 5.2. The second set has zero measure since $(\{z\} \times$ $\mathbb{R}) \cap J_{u}$ was at most countable. In total, $\mathcal{L}^{1}(M u(z, N))=0$, and so $M u$ has the Lusin property on almost every line parallel to a coordinate axis.

If we could give a positive answer to the following open problem, then we could extend Theorem 5.4 from SBV functions to BV functions.

Open Problem. Let $u \in \mathrm{BV}\left(\mathbb{R}^{d}\right)$. Is it true that $M u(x)>|u|^{\vee}(x)$ for $\left|D^{c} u\right|$-almost every $x \in \mathbb{R}^{d}$ ?

In one dimension the answer is yes, see Proposition 8.1.

\section{Sobolev property}

In this section we show that if the non-centered maximal function of an SBV function is locally BV, then it is in fact locally Sobolev.

We rely on the following direction of the Banach-Zarecki Theorem. 
Theorem 6.1. Let $V \subset \mathbb{R}$ be open and let $v \in \mathrm{BV}_{\text {loc }}(V)$ be a continuous function that satisfies the Lusin property. Then $v$ is absolutely continuous on $V$.

We say that a function $v$ on $\mathbb{R}^{d}$ is ACL if it is absolutely continuous on almost every line parallel to a coordinate axis.

The following theorem is Theorem 1.2 in a slightly more general form.

Theorem 6.2. Let $u \in \mathrm{SBV}_{\text {loc }}\left(\mathbb{R}^{d}\right) \cap L^{1}\left(\mathbb{R}^{d}\right)$ or $u \in \mathrm{SBV}_{\text {loc }}\left(\mathbb{R}^{d}\right) \cap L^{\infty}\left(\mathbb{R}^{d}\right)$. If $M u \in \mathrm{BV}_{\mathrm{loc}}\left(\mathbb{R}^{d}\right)$, then $M u$ is $A C L$ and in the class $W_{\mathrm{loc}}^{1,1}\left(\mathbb{R}^{d}\right)$.

Proof. It is sufficient to consider the $d$ :th coordinate direction. Since $M u \in \mathrm{BV}_{\text {loc }}\left(\mathbb{R}^{d}\right)$, the function $M u(z, \cdot)$ is in the class $\mathrm{BV}_{\text {loc }}(\mathbb{R})$ for almost every $z \in \mathbb{R}^{d-1}$. By Theorem $5.4, M u(z, \cdot)$ is also continuous and has the Lusin property for almost every $z \in \mathbb{R}^{d-1}$. Now by the Banach-Zarecki theorem, $M u(z, \cdot)$ is absolutely continuous on $\mathbb{R}$ for almost every $z \in \mathbb{R}^{d-1}$. In conclusion, $M u$ is ACL. Since we already know that $M u \in \mathrm{BV}_{\text {loc }}\left(\mathbb{R}^{d}\right)$, it follows that $M u \in W_{\text {loc }}^{1,1}\left(\mathbb{R}^{d}\right)$.

In Theorem 6.2, we would of course like to prove that $M u \in \mathrm{BV}_{\text {loc }}\left(\mathbb{R}^{d}\right)$, instead of assuming this. For sets of finite perimeter, such a better result is possible due to a very recent result of Weigt [12]. We restate Theorem 1.3, which is our third main theorem:

Theorem 6.3. Let $E \subset \mathbb{R}^{d}$ be a set of finite perimeter. Then $M \mathbb{1}_{E} \in W_{\text {loc }}^{1,1}\left(\mathbb{R}^{d}\right)$ with $\left\|\nabla M \mathbb{1}_{E}\right\|_{L^{1}\left(\mathbb{R}^{d}\right)} \leq C_{d}\left|D \mathbb{1}_{E}\right|\left(\mathbb{R}^{d}\right)$, where $C_{d}$ only depends on the dimension $d$.

Proof. Theorem 1.3 of $[12]$ states that $M \mathbb{1}_{E} \in \mathrm{BV}_{\text {loc }}\left(\mathbb{R}^{d}\right)$ with $\left|D M \mathbb{1}_{E}\right|\left(\mathbb{R}^{d}\right) \leq$ $C_{d}\left|D \mathbb{1}_{E}\right|\left(\mathbb{R}^{d}\right)$. Then by Theorem 6.2 , the conclusion follows.

\section{Formula for the gradient}

When $u \in \operatorname{BV}(\Omega)$ and $M_{\Omega} u \in W_{\text {loc }}^{1,1}(\Omega)$, there is a weak gradient $D M_{\Omega} u \in L_{\text {loc }}^{1}(\Omega)$. In this section we derive a formula for it, generalizing [10, Lemma 2.2(1)] where the case $u \in W^{1,1}\left(\mathbb{R}^{d}\right)$ was considered. However, as we will see, in the case $u \in \operatorname{BV}(\Omega)$ the formula is, and can be, valid only under certain conditions.

As usual, $\Omega \subset \mathbb{R}^{d}$ is an arbitrary nonempty open set.

We have the following standard approximation result for BV functions.

Proposition 7.1. Let $u \in \mathrm{BV}(\Omega)$. Then there exists a sequence of functions $\left\{v_{i}\right\}_{i \in \mathbb{N}}$ in $C^{\infty}(\Omega)$ such that $v_{i} \rightarrow u$ in $L^{1}(\Omega), \lim _{i \rightarrow \infty}\left|D v_{i}\right|(\Omega)=|D u|(\Omega)$, and $D v_{i} \stackrel{*}{\rightarrow} D u$ and $\left|D v_{i}\right| \stackrel{*}{\rightarrow}|D u|$ in $\Omega$.

Proof. By [3, Theorem 3.9] we find a sequence of functions $\left\{v_{i}\right\}_{i \in \mathbb{N}}$ in $C^{\infty}(\Omega)$ such that $v_{i} \rightarrow u$ in $L^{1}(\Omega)$ and $\lim _{i \rightarrow \infty}\left|D v_{i}\right|(\Omega)=|D u|(\Omega)$. Then by [3, Proposition 3.13] we have in fact $D v_{i} \stackrel{*}{\rightarrow} D u$ in $\Omega$, and by [3, Proposition 1.80] also $\left|D v_{i}\right| \stackrel{*}{\rightarrow}|D u|$ in $\Omega$.

We also have the following simple fact concerning the measures of spheres. 
Lemma 7.2. Let $\nu$ be a positive Radon measure on $\Omega$ with $\nu(\Omega)<\infty$ and $\nu \ll \mathcal{H}^{d-1}$. Then $\nu(\partial B)>0$ for at most countably many spheres $\partial B$ with $\bar{B} \subset \Omega$.

Proof. For any distinct $y, z \in \Omega$ with $\bar{B}(y, r) \subset \Omega$ and $\bar{B}(z, R) \subset \Omega$ for some $r, R>0$, the intersection of the spheres $\partial B(y, r)$ and $\partial B(z, R)$ has zero $\mathcal{H}^{d-1}$-measure and thus zero $\nu$-measure. Thus for every $\alpha>0$, there can be only finitely many balls $\bar{B} \subset \Omega$ such that $\nu(\partial B)>\alpha$. The result follows.

Given a ball $B=B(x, r)$ (open, as usual) and $k \in\{1, \ldots, d\}$, define the "halfopen" balls

$$
\bar{B}^{k,+}:=B \cup\left\{y \in \partial B: y_{k}>x_{k}\right\}, \quad \bar{B}^{k,-}:=B \cup\left\{y \in \partial B: y_{k}<x_{k}\right\} .
$$

The following definition of "optimal balls" is convenient when studying the noncentered maximal function, and has been used e.g. in [10]. Recall also Lemma 3.1 .

Definition 7.3. For a function $u$ on $\Omega$ and $x \in \Omega$, let

$$
\mathcal{B}_{x}:=\left\{B(z, r) \subset \Omega: x \in \bar{B}(z, r), r>0, \text { and } f_{B(z, r)}|u| d y=M_{\Omega} u(x)\right\} .
$$

Note that if $u \in L^{1}(\Omega)$ and $x \in \Omega$ is a Lebesgue point of $u$ with $\mathcal{B}_{x}=\emptyset$, then we necessarily have $M_{\Omega} u(x)=\widetilde{u}(x)$.

The following is our first version of a formula for $D M_{\Omega} u$. Note that when $M_{\Omega} u \in$ $\mathrm{BV}_{\text {loc }}(\Omega)$, at almost every $x \in \Omega$ we can interpret $D_{k} M_{\Omega} u(x), k \in\{1, \ldots, d\}$, to be either the density of the measure $D_{k} M_{\Omega} u$ or the classical partial derivative of $M_{\Omega} u$ (for the classical partial derivative to make sense, we need the correct pointwise representative of $M_{\Omega} u$, but the first part of Theorem 5.4 guarantees that $M_{\Omega} u$ itself is suitable). The same applies to $D_{k} \widetilde{u}$.

Theorem 7.4. Let $u \in \mathrm{BV}(\Omega)$ such that $M_{\Omega} u \in \mathrm{BV}_{\text {loc }}(\Omega)$. Then for almost every $x \in \Omega$ and every $k \in\{1, \ldots, d\}$, we have

$$
\begin{aligned}
& \text { (1) } \frac{D_{k}|u|\left(\bar{B}^{k,+}\right)}{\mathcal{L}^{d}(B)} \leq D_{k} M_{\Omega} u(x) \leq \frac{D_{k}|u|\left(\bar{B}^{k,-}\right)}{\mathcal{L}^{d}(B)} \quad \text { if } B \in \mathcal{B}_{x}, \bar{B} \subset \Omega, \\
& \text { (2) } D_{k} M_{\Omega} u(x)=D_{k}|\widetilde{u}|(x) \quad \text { if } \mathcal{B}_{x}=\emptyset
\end{aligned}
$$

Proof. As usual, we can assume that $u \geq 0$. Take $x \in \Omega$ such that all $D_{k} M_{\Omega} u(x)$, $k=1, \ldots, d$, exist (both as densities and as classical derivatives).

First suppose that $B(z, r) \in \mathcal{B}_{x}$ and $\overline{B(z, r)} \subset \Omega$. Fix $k \in\{1, \ldots, d\}$. Now $B\left(z+h e_{k}, r\right) \subset \Omega$ for $h \in \mathbb{R}$ close to zero. 
Consider momentarily $v \in C^{\infty}(\Omega)$. We get for small $h>0$ that

$$
\begin{aligned}
\frac{1}{h}\left(f_{B\left(z+h e_{k}, r\right)} v d y-f_{B(z, r)} v d y\right) & =\frac{1}{h}\left(f_{B(z, r)} v\left(y+h e_{k}\right)-v(y) d y\right) \\
& =\frac{1}{h}\left(f_{B(z, r)} \int_{0}^{h} D_{k} v\left(y+t e_{k}\right) d t d y\right) \\
& =\frac{1}{h} \int_{0}^{h}\left(f_{B\left(z+t e_{k}, r\right)} D_{k} v d y\right) d t
\end{aligned}
$$

By Proposition 7.1 we find a sequence $\left\{v_{i}\right\}_{i \in \mathbb{N}}$ in $C^{\infty}(\Omega)$ such that $v_{i} \rightarrow u$ in $L^{1}(\Omega)$, $\lim _{i \rightarrow \infty}\left|D v_{i}\right|(\Omega)=|D u|(\Omega)$, and $D v_{i} \stackrel{*}{\rightarrow} D u$ and $\left|D v_{i}\right| \stackrel{*}{\rightarrow}|D u|$ in $\Omega$. For every ball $B$ with $\bar{B} \subset \Omega$ and $|D u|(\partial B)=0$, this implies (see [3, Proposition 1.62(b)])

$$
D v_{i}(B) \rightarrow D u(B) \quad \text { so in particular } \quad D_{k} v_{i}(B) \rightarrow D_{k} u(B) .
$$

By Lemma 7.2, there are at most countably many spheres $\partial B$ with $\bar{B} \subset \Omega$ and $|D u|(\partial B)>0$. In particular, $|D u|\left(\partial B\left(z+t e_{k}, r\right)\right)=0$ for almost every $t \in[0, h]$. Writing (7.5) with $v=v_{i}$ and taking the limit $i \rightarrow \infty$, we get by Lebesgue's dominated convergence theorem

$$
\frac{1}{h}\left(f_{B\left(z+h e_{k}, r\right)} u d y-f_{B(z, r)} u d y\right)=\frac{1}{h} \int_{0}^{h}\left(\frac{D_{k} u\left(B\left(z+t e_{k}, r\right)\right)}{\mathcal{L}^{d}(B(z, r))}\right) d t
$$

Thus

$$
\begin{aligned}
D_{k} M_{\Omega} u(x) & =\lim _{h \rightarrow 0^{+}} \frac{1}{h}\left(M_{\Omega} u\left(x+h e_{k}\right)-M_{\Omega} u(x)\right) \\
& \geq \lim _{h \rightarrow 0^{+}} \frac{1}{h}\left(f_{B\left(z+h e_{k}, r\right)} u d y-f_{B(z, r)} u d y\right) \\
& =\lim _{h \rightarrow 0^{+}} \frac{1}{h} \int_{0}^{h}\left(\frac{D_{k} u\left(B\left(z+t e_{k}, r\right)\right)}{\mathcal{L}^{d}(B(z, r))}\right) d t .
\end{aligned}
$$

Here

$$
\begin{aligned}
& \left|\frac{1}{h} \int_{0}^{h}\left(\frac{D_{k} u\left(B\left(z+t e_{k}, r\right)\right)}{\mathcal{L}^{d}(B(z, r))}\right) d t-\frac{D_{k} u\left(\bar{B}^{k,+}(z, r)\right)}{\mathcal{L}^{d}(B(z, r))}\right| \\
& \leq \frac{1}{h} \int_{0}^{h}\left|\frac{D_{k} u\left(B\left(z+t e_{k}, r\right)\right)}{\mathcal{L}^{d}(B(z, r))}-\frac{D_{k} u\left(\bar{B}^{k,+}(z, r)\right)}{\mathcal{L}^{d}(B(z, r))}\right| d t \\
& \leq \frac{1}{\mathcal{L}^{d}(B(z, r))} \frac{1}{h} \int_{0}^{h}|D u|\left(\bar{B}^{k,+}(z, r) \Delta \bigcup_{s \in(0, h)} B\left(z+s e_{k}, r\right)\right) d t \\
& =\frac{1}{\mathcal{L}^{d}(B(z, r))}|D u|\left(\bar{B}^{k,+}(z, r) \Delta \bigcup_{s \in(0, h)} B\left(z+s e_{k}, r\right)\right) \\
& \rightarrow 0 \quad \text { as } h \rightarrow 0
\end{aligned}
$$


since $\bar{B}^{k,+}(z, r) \Delta \bigcup_{s \in(0, h)} B\left(z+s e_{k}, r\right) \rightarrow \emptyset$. Combining the previous inequalities, we get

$$
D_{k} M_{\Omega} u(x) \geq \frac{D_{k} u\left(\bar{B}^{k,+}(z, r)\right)}{\mathcal{L}^{d}(B(z, r))} .
$$

Similarly we get

$$
\begin{aligned}
D_{k} M_{\Omega} u(x) & =\lim _{h \rightarrow 0^{+}} \frac{1}{h}\left(M_{\Omega} u(x)-M_{\Omega} u\left(x-h e_{k}\right)\right) \\
& \leq \lim _{h \rightarrow 0^{+}} \frac{1}{h}\left(f_{B(z, r)} u d y-f_{B\left(z-h e_{k}, r\right)} u d y\right) \\
& =\frac{D_{k} u\left(\bar{B}^{k,-}(z, r)\right)}{\mathcal{L}^{d}(B(z, r))} .
\end{aligned}
$$

Then assume that $\mathcal{B}_{x}=\emptyset$. Discarding another $\mathcal{L}^{d}$-negligible set, we can assume that $x$ is a Lebesgue point of $u$, and so necessarily $M_{\Omega} u(x)=\widetilde{u}(x)$. We can also assume that $D_{k} \widetilde{u}(x), k=1, \ldots, d$, exist (again, both as densities and as partial derivatives). Thus

$$
\begin{aligned}
D_{k} M_{\Omega} u(x) & =\lim _{h \rightarrow 0^{+}} \frac{1}{h}\left(M_{\Omega} u\left(x+h e_{k}\right)-M_{\Omega} u(x)\right) \\
& \geq \lim _{h \rightarrow 0^{+}} \frac{1}{h}\left(\widetilde{u}\left(x+h e_{k}\right)-\widetilde{u}(x)\right) \\
& =D_{k} \widetilde{u}(x) .
\end{aligned}
$$

Similarly,

$$
\begin{aligned}
D_{k} M_{\Omega} u(x) & =\lim _{h \rightarrow 0^{+}} \frac{1}{h}\left(M_{\Omega} u(x)-M_{\Omega} u\left(x-h e_{k}\right)\right) \\
& \leq \lim _{h \rightarrow 0^{+}} \frac{1}{h}\left(\widetilde{u}\left(x+h e_{k}\right)-\widetilde{u}(x)\right) \\
& =D_{k} \widetilde{u}(x) .
\end{aligned}
$$

From the viewpoint of having a formula for $D M_{\Omega} u$, we would of course like to have equality in Theorem 7.4(1), which in particular happens if $|D u|(\partial B)=0$. With this in mind, we prove the following lemma.

Lemma 7.6. Let $d \geq 2$ and let $u \in \mathrm{BV}_{\text {loc }}(\Omega)$. For $\mathcal{L}^{d}$-almost every $x \in \Omega$, we have

$$
|D u|(\partial B)=0
$$

for every ball $B$ with $\bar{B} \subset \Omega$ and $x \in \partial B$.

Proof. We can apply Lemma 7.2 with the choice $\nu=|D u|$ to obtain that there are at most countably many spheres $\partial B$ such that $|D u|(\partial B)>0$. Thus $|D u|(\partial B)>0$ and $x \in \partial B$ can only be true if $x$ belongs to the countable union of spheres, and such a union of course has Lebesgue measure zero. 
The following fact is easy to prove, see [10, Lemma 2.2(2)].

Lemma 7.7. Let $u$ be a function on $\Omega$ and let $x \in \Omega$. If there is a ball $B \in \mathcal{B}_{x}$ with $x \in B$, then $D M_{\Omega} u(x)=0$ (as a classical derivative).

Now we can prove the following formula for the gradient of the maximal function.

Theorem 7.8. Let $u \in \mathrm{BV}(\Omega)$ with $M_{\Omega} u \in \mathrm{BV}_{\text {loc }}(\Omega)$. Then for almost every $x \in \Omega$,

(1) $D M_{\Omega} u(x)=\frac{D|u|(B)}{\mathcal{L}^{d}(B)} \quad$ if $B \in \mathcal{B}_{x}, \bar{B} \subset \Omega, D M_{\Omega} u(x) \neq 0$, and $d \geq 2$,

(2) $D M_{\Omega} u(x)=D|\widetilde{u}|(x) \quad$ if $\mathcal{B}_{x}=\emptyset$.

In Examples 7.9 and 7.10 we will show that the assumptions $\bar{B} \subset \Omega, D M_{\Omega} u(x) \neq$ 0 , and $d \geq 2$ are needed.

Proof. First suppose that $B \in \mathcal{B}_{x}, \bar{B} \subset \Omega, D M_{\Omega} u(x) \neq 0$, and $d \geq 2$. Note that since $D M_{\Omega} u(x) \neq 0$, we have $x \in \partial B$ by Lemma 7.7. Then by Lemma 7.6 we can assume that $|D u|(\partial B)=0$ (recall (2.6)). Thus by Theorem 7.4(1), we get (1).

If $\mathcal{B}_{x}=\emptyset$, Theorem $7.4(2)$ gives (2).

Example 7.9. On the real line, take $\Omega=\mathbb{R}$ and

$$
u(x):= \begin{cases}x & \text { when } 0 \leq x \leq 1 \\ 0 & \text { otherwise }\end{cases}
$$

Thus $u \in \mathrm{BV}(\mathbb{R})$. Now obviously for every $x \in[0,1]$ we have $\mathcal{B}_{x}=\{(x, 1)\}$, so that

$$
M u(x)=f_{(x, 1)} u(t) d t=\frac{1}{2}(1+x) .
$$

Hence $D M u(x)=1 / 2$ for all $x \in(0,1)$, but

$$
\frac{D u((x, 1))}{\mathcal{L}^{1}((x, 1))}=\frac{D u([x, 1))}{\mathcal{L}^{1}((x, 1))}=1 \quad \text { and } \quad \frac{D u((x, 1])}{\mathcal{L}^{1}((x, 1))}=\frac{D u([x, 1])}{\mathcal{L}^{1}((x, 1))}=1-\frac{1}{1-x} .
$$

Thus Theorem 7.8(1) fails, also if $B$ is replaced by any half-open interval. Hence the assumption $d \geq 2$ is necessary.

A small modification of this example shows that the assumption $\bar{B} \subset \Omega$ is also needed. On the plane, let $\Omega=(0,1) \times(-2,2)$ and

$$
u\left(x_{1}, x_{2}\right):=x_{1} .
$$

Now obviously for every $x \in(0,1) \times(-1,1)$, we have $\mathcal{B}_{x}=\left\{B_{x}\right\}$ with

$$
B_{x}:=\left\{B\left(\left(\frac{1+x_{1}}{2}, x_{2}\right), \frac{1-x_{1}}{2}\right)\right\},
$$

so that

$$
M_{\Omega} u(x)=f_{B_{x}} u d y=\frac{1}{2}\left(1+x_{1}\right) .
$$


Hence $D_{1} M_{\Omega} u(x)=1 / 2$ for all $x \in(0,1) \times(-1,1)$, but

$$
\frac{D_{1} u\left(B_{x}\right)}{\mathcal{L}^{2}\left(B_{x}\right)}=1
$$

showing that the assumption $\bar{B} \subset \Omega$ is necessary. Moreover $|D u|\left(\partial B_{x} \backslash \partial \Omega\right)=0$, so again including any part of the boundary of $B_{x}$ would not help either.

Finally we give an example showing that the assumption $D M_{\Omega} u(x) \neq 0$ is also needed in Theorem 7.8.

Example 7.10. On the plane, let $\Omega=\mathbb{R}^{2}$ and

$$
E_{0}:=B(0,1) \backslash B(0,1-\delta)
$$

for a small $\delta$; choose $\delta=0.01$. Then for every $x \in B(0, \delta)$, we claim that $\mathcal{B}_{x}^{E_{0}}=$ $\{B(0,1)\}$; we use the superscript $E_{0}$ to specify that we consider the collection of optimal balls with respect to the function $\mathbb{1}_{E_{0}}$. To see this, fix $x \in B(0, \delta)$ and $B(z, r) \in \mathcal{B}_{x}^{E_{0}}$ (such a disk is easily seen to exist). Note first that necessarily $0.49 \leq$ $r \leq 1$. Now we simply check three cases.

First suppose $0.49 \leq r \leq 0.63$. Now $B(z, r)$ intersects less than $22 / 100$ of $\partial B(0,1-\delta)$, and so

$$
\begin{aligned}
m(B(z, r)) & :=\frac{\mathcal{L}^{2}\left(E_{0} \cap B(z, r)\right)}{\mathcal{L}^{2}(B(z, r))} \leq \frac{\mathcal{L}^{2}\left(E_{0} \cap B(z, 0.63)\right)}{\mathcal{L}^{2}(B(z, 0.49))} \\
& <\frac{\frac{22}{100} \times 2 \pi \delta}{0.49^{2} \mathcal{L}^{2}(B(0,1))}<\frac{0.99 \times 2 \pi \delta}{\mathcal{L}^{2}(B(0,1))}<\frac{\mathcal{L}^{2}\left(E_{0}\right)}{\mathcal{L}^{2}(B(0,1))}=m(B(0,1)) .
\end{aligned}
$$

Then suppose $0.63 \leq r \leq 0.8$. Now $B(z, r)$ intersects less than $1 / 3$ of $\partial B(0,1-\delta)$, and so

$$
m(B(z, r))<\frac{\frac{1}{3} \times 2 \pi \delta}{0.63^{2} \mathcal{L}^{2}(B(0,1))}<m(B(0,1)) .
$$

Finally suppose $0.8 \leq r \leq 0.95$. Now $B(z, r)$ intersects less than $1 / 2$ of $\partial B(0,1-\delta)$, and so

$$
m(B(z, r))<\frac{\frac{1}{2} \times 2 \pi \delta}{0.8^{2} \mathcal{L}^{2}(B(0,1))}<m(B(0,1)) .
$$

Thus necessarily $0.95 \leq r \leq 1$. But now $B(z, r) \supset \partial B(0,1-\delta)$, because otherwise $B(z, r)$ covers less than $3 / 4$ of $\partial B(0,1-\delta / 2)$, implying that

$$
m(B(z, r))<\frac{\frac{7}{8} \times 2 \pi \delta}{0.95^{2} \mathcal{L}^{2}(B(0,1))}<m(B(0,1)) .
$$

Thus $B(z, r)$ contains $B(0,1-\delta)$, and now clearly the maximum value of $m(B(z, r))$ is obtained by choosing $B(z, r)=B(0,1)$. Thus $\mathcal{B}_{x}^{E_{0}}=\{B(0,1)\}$ as desired. 
Denote $c:=(0,1) \in \partial B(0,1)$. Next we "perturb" $E_{0}$ slightly by removing and adding a small ball:

$$
E:=\left(E_{0} \backslash B\left(c, \delta^{2}\right)\right) \cup\left(B\left(c, \delta^{2}\right) \backslash B(0,1)\right) .
$$

Since the perturbation is so small, almost the same calculations as above show that for every $x \in B(0, \delta)$, we have $\mathcal{B}_{x}^{E}=\{B(0,1)\}$. Thus $D M \mathbb{1}_{E}=0$ in $B(0, \delta)$, but

$$
\begin{gathered}
\frac{D_{2} \mathbb{1}_{E}(B(0,1))}{\mathcal{L}^{2}(B(0,1))}<0, \quad \frac{D_{2} \mathbb{1}_{E}(\bar{B}(0,1))}{\mathcal{L}^{2}(B(0,1))}>0, \\
\frac{D_{2} \mathbb{1}_{E}\left(\bar{B}^{2,+}(0,1)\right)}{\mathcal{L}^{2}(B(0,1))}<0, \quad \frac{D_{2} \mathbb{1}_{E}\left(\bar{B}^{2,-}(0,1)\right)}{\mathcal{L}^{2}(B(0,1))}>0 .
\end{gathered}
$$

Thus none of these equal $D M \mathbb{1}_{E}$ in $B(0, \delta)$, which is of course a set of nonzero Lebesgue measure.

\section{The one-dimensional case}

In this section we investigate the properties of the non-centered maximal function in the special case $d=1$. Let $\Omega \subset \mathbb{R}$ be an arbitrary nonempty open set.

Aldaz and Pérez Lázaro [1] proved in one dimension that the non-centered maximal function of a function $u \in \operatorname{BV}(\Omega)$ is in the Sobolev class $W_{\text {loc }}^{1,1}(\Omega)$, with $\left\|D M_{\Omega} u\right\|_{L^{1}(\Omega)} \leq|D u|(\Omega)$. Now we investigate the behavior of $M_{\Omega} u$ a little further.

Recall that

$$
H_{u}=\left\{x \in \Omega: M_{\Omega} u(x)>|u|^{\vee}(x)\right\} .
$$

Recall also from the proof of Theorem 5.4 and the Open Problem on Page 15 that it is in some sense desirable that the set $H_{u}$ be as large as possible. On the real line, we are able to show the following. Recall that $S_{u}$ is the set of non-Lebesgue points, or (as we are in one dimension) the set of discontinuity points of $u^{\vee}$ (alternatively some other good pointwise representative). Moreover, denote by $\partial^{*} E$ the measuretheoretic boundary of a set $E \subset \mathbb{R}$, i.e. the set of points $x \in \mathbb{R}$ for which

$$
\limsup _{r \rightarrow 0} \frac{\mathcal{L}^{1}(B(x, r) \cap E)}{\mathcal{L}^{1}(B(x, r))}>0 \quad \text { and } \quad \limsup _{r \rightarrow 0} \frac{\mathcal{L}^{1}(B(x, r) \backslash E)}{\mathcal{L}^{1}(B(x, r))}>0 .
$$

Proposition 8.1. Let $u \in \mathrm{BV}_{\text {loc }}(\Omega)$ with $|D u|(\Omega)<\infty$. Then $|D u|\left(\Omega \backslash\left(H_{u} \cup S_{u}\right)\right)=$ 0 .

Proof. Abbreviate super-level sets by

$$
\{u>t\}:=\{x \in \Omega: u(x)>t\}, \quad t \in \mathbb{R} .
$$

We have the coarea formula (see e.g. [3, Theorem 3.40])

$$
|D u|(A)=\int_{-\infty}^{\infty}\left|D \mathbb{1}_{\{u>t\}}\right|(A) d t
$$


for every Borel set $A \subset \Omega$. In particular, for almost every $t \in \mathbb{R}$, the super-level set $\{u>t\}$ has finite perimeter in $\Omega$. Let $N \subset \mathbb{R}$ be the exceptional set. For later purposes, we also include in $N$ the at most countably many $t \in \mathbb{R}$ for which $\mathcal{L}^{1}(\{u=t\})>0$.

Consider $t \in \mathbb{R} \backslash N$. First assume that $t \geq 0$. The fact that $\{u>t\}$ has finite perimeter in $\Omega$ means that after redefinition in a set of measure zero, giving a set that we can denote $E_{t}$, the relative boundary $\partial E_{t} \cap \Omega \subset \Omega$ consists of finitely many points; see [3, Proposition 3.52]. It follows that if $x \in \partial E_{t} \cap \Omega$, then for small enough $r>0$, necessarily $\mathcal{L}^{1}((x-r, x) \backslash\{u \leq t\})=0$ and $\mathcal{L}^{1}((x, x+r) \backslash\{u>t\})=0$, or vice versa. Supposing without loss of generality the former, we have

$$
M_{\Omega} u(x) \geq f_{(x, x+r)} u d y>t .
$$

If also $x \notin S_{u}$, then $t=u^{\vee}(x)=|u|^{\vee}(x)$, and we get $x \in H_{u}$. In conclusion, if $x \in \partial^{*}\{u>t\} \cap \Omega \backslash S_{u} \subset \partial E_{t} \cap \Omega \backslash S_{u}$ for some $t \in \mathbb{R} \backslash N$, then $x \in H_{u}$.

For $t<0$, we similarly get that if $x \in \partial E_{t} \cap \Omega$ for $t \in \mathbb{R} \backslash N$, then necessarily $\mathcal{L}^{1}((x-r, x) \backslash\{u<t\})=0$ and $\mathcal{L}^{1}((x, x+r) \backslash\{u>t\})=0$, or vice versa (recall that $\left.\mathcal{L}^{1}(\{u=t\})=0\right)$. Supposing the former,

$$
M_{\Omega} u(x) \geq f_{(x-r, x)}|u| d y>|t| .
$$

If $x \notin S_{u}$, then $|t|=|u|^{\vee}(x)$ and we get $x \in H_{u}$, as before.

Consider a point $x$ in the Borel set $A:=\Omega \backslash\left(H_{u} \cup S_{u}\right)$. Now $x \notin \partial^{*}\{u>t\}$ for all $t \in \mathbb{R} \backslash N$. For every $t \in \mathbb{R} \backslash N$ we also have

$$
\left|D \mathbb{1}_{\{u>t\}}\right|(A)=\mathcal{H}^{0}\left(\partial^{*}\{u>t\} \cap A\right),
$$

see e.g. [3, Theorems $3.59 \& 3.61]$. Thus by $(8.2)$ we get

$$
|D u|(A)=\int_{-\infty}^{\infty} \mathcal{H}^{0}\left(\partial^{*}\{u>t\} \cap A\right) d t=\int_{N} \mathcal{H}^{0}\left(\partial^{*}\{u>t\} \cap A\right) d t=0 .
$$

Since $M_{\Omega} u$ is continuous (even absolutely) and $u^{\vee}$ is upper semicontinuous, $H_{u}$ is an open set, so it is the union of disjoint open intervals

$$
H_{u}=\bigcup_{j=1}^{\infty}\left(a_{j}, b_{j}\right) \subset \Omega .
$$

Note that for two of these intervals, $a_{j}$ or $b_{j}$ may be $\pm \infty$.

Theorem 8.4. Let $u \in \mathrm{BV}_{\text {loc }}(\Omega)$ with $|D u|(\Omega)<\infty$. For each $j \in \mathbb{N}$ there exists a point $c_{j} \in\left(a_{j}, b_{j}\right)$ such that we have the representation

$$
\left|D M_{\Omega} u\right|(\Omega)=\sum_{j=1}^{\infty}\left[\left|M_{\Omega} u\left(a_{j}\right)-M_{\Omega} u\left(c_{j}\right)\right|+\left|M_{\Omega} u\left(c_{j}\right)-M_{\Omega} u\left(b_{j}\right)\right|\right] .
$$


If $a_{j}$ (or $b_{j}$ ) is $\pm \infty$, we interpret $M_{\Omega} u\left(a_{j}\right)$ as a limit; it exists since $M_{\Omega} u \in$ $W_{\text {loc }}^{1,1}(\Omega)$ with $\left\|D M_{\Omega} u\right\|_{L^{1}(\Omega)} \leq|D u|(\Omega)$ by $[1$, Theorem 2.5$]$.

Proof. The set $S_{u}$ is at most countable and $M_{\Omega} u \in W_{\text {loc }}^{1,1}(\Omega)$, so we have

$$
\left|D M_{\Omega} u\right|\left(S_{u}\right)=0 .
$$

Consider a point $x \in \Omega \backslash\left(S_{u} \cup H_{u}\right)$. Now $M_{\Omega} u(x)=|u|^{\vee}(x)$. Since both functions $M_{\Omega} u$ and $|u|^{\vee}$ are continuous at $x$, this point can be in $\partial^{*}\left\{M_{\Omega} u-|u|>t\right\}$ only for $t=0$. By the coarea formula (8.2) and (8.3), we have

$$
\begin{aligned}
\left|D\left(M_{\Omega} u-|u|\right)\right|\left(\Omega \backslash\left(S_{u} \cup H_{u}\right)\right) & =\int_{-\infty}^{\infty} \mathcal{H}^{0}\left(\Omega \cap \partial^{*}\left\{M_{\Omega} u-|u|>t\right\} \backslash\left(S_{u} \cup H_{u}\right)\right) d t \\
& =0 .
\end{aligned}
$$

By Proposition 8.1, it follows that

$$
\left|D M_{\Omega} u\right|\left(\Omega \backslash\left(S_{u} \cup H_{u}\right)\right)=|D| u||\left(\Omega \backslash\left(S_{u} \cup H_{u}\right)\right) \leq|D u|\left(\Omega \backslash\left(S_{u} \cup H_{u}\right)\right)=0 .
$$

Thus by (8.5),

$$
\left|D M_{\Omega} u\right|\left(\Omega \backslash H_{u}\right)=0 .
$$

Thus all of the total variation of $M_{\Omega} u$ is in the set $H_{u}=\bigcup_{j=1}^{\infty}\left(a_{j}, b_{j}\right)$.

Now we follow an argument given in [1]. Suppose that for some $j \in \mathbb{N}$, there exist points $d_{1}, d_{2}, d_{3}$ with $a_{j}<d_{1}<d_{2}<d_{3}<b_{j}$ and $M_{\Omega} u\left(d_{1}\right)<M_{\Omega} u\left(d_{2}\right)$ and $M_{\Omega} u\left(d_{3}\right)<M_{\Omega} u\left(d_{2}\right)$. We can assume that $M_{\Omega} u\left(d_{2}\right)=\max \left\{M_{\Omega} u(x): x \in\left[d_{1}, d_{3}\right]\right\}$. Then by [1, Lemma 3.6] we have $M_{\Omega} u\left(d_{2}\right)=|u|^{\vee}\left(d_{2}\right)$, a contradiction with $d_{2} \in H_{u}$.

It follows that for every $j \in \mathbb{N}$, either $M_{\Omega} u$ is monotone on $\left(a_{j}, b_{j}\right)$ or there exists $c_{j} \in\left(a_{j}, b_{j}\right)$ such that $M_{\Omega} u$ is decreasing on $\left[a_{j}, c_{j}\right]$ and increasing on $\left[c_{j}, b_{j}\right]$. In the former case, we can just choose an arbitrary $c_{j} \in\left(a_{j}, b_{j}\right)$. Now

$$
\left|D M_{\Omega} u\right|(\Omega)=\left|D M_{\Omega} u\right|\left(H_{u}\right)=\sum_{j=1}^{\infty}\left[\left|M_{\Omega} u\left(a_{j}\right)-M_{\Omega} u\left(c_{j}\right)\right|+\left|M_{\Omega} u\left(c_{j}\right)-M_{\Omega} u\left(b_{j}\right)\right|\right] .
$$

\section{References}

[1] J. M. Aldaz and F. J. Pérez Lázaro, Functions of bounded variation, the derivative of the one dimensional maximal function, and applications to inequalities, Trans. Amer. Math. Soc. 359 (2007), no. 5, 2443-2461. 2, 7, 22,24

[2] J. M. Aldaz and F. J. Pérez Lázaro, Regularity of the Hardy-Littlewood maximal operator on block decreasing functions, Studia Math. 194 (2009), no. 3, 253-277. 1, 2, 12 
[3] L. Ambrosio, N. Fusco, and D. Pallara, Functions of bounded variation and free discontinuity problems. Oxford Mathematical Monographs. The Clarendon Press, Oxford University Press, New York, 2000. 4, 5, 15, 16 , $18,22,23$

[4] M. Carriero, G. Dal Maso, A. Leaci, and E. Pascali, Relaxation of the nonparametric plateau problem with an obstacle, J. Math. Pures Appl. (9) 67 (1988), no. 4, 359-396. 7

[5] H. Hakkarainen and J. Kinnunen, The BV-capacity in metric spaces, Manuscripta Math. 132 (2010), no. 1-2, 51-73. 3

[6] J. Kinnunen, The Hardy-Littlewood maximal function of a Sobolev function, Israel J. Math. 100 (1997), 117-124. 1

[7] J. Kinnunen, R. Korte, N. Shanmugalingam, and H. Tuominen, Lebesgue points and capacities via the boxing inequality in metric spaces, Indiana Univ. Math. J. 57 (2008), no. 1, 401-430. 7

[8] P. Lahti, Strong approximation of sets of finite perimeter in metric spaces, Manuscripta Math. 155 (2018), no. 3-4, 503-522. 7, 8

[9] P. Lahti and N. Shanmugalingam, Fine properties and a notion of quasicontinuity for BV functions on metric spaces, J. Math. Pures Appl. (9) 107 (2017), no. 2, 150-182. 7

[10] H. Luiro, The variation of the maximal function of a radial function, Ark. Mat. 56 (2018), no. 1, 147-161. 1, 6, 7, 16, 17, 20

[11] H. Tanaka, A remark on the derivative of the one-dimensional HardyLittlewood maximal function, Bull. Austral. Math. Soc. 65 (2002), no. 2, 253-258. 1

[12] J. Weigt, Variation of the uncentered maximal characteristic function, preprint 2020.

https://arxiv.org/abs/2004.10485 1, 2, 16

Address:

Academy of Mathematics and Systems Science, Chinese Academy of Sciences,

Beijing 100190, PR China

E-mail: panu.lahti@aalto.fi 